\title{
Echocardiographic Profile of Human Immunodeficiency Virus (HIV) Infected Patients in a Tertiary Care Hospital.
}

\author{
Padhiyar Rupal N*, Chavan Swati A**. \\ * Assistant Professor Of Medicine, \\ ** Professor (Addl) Of Medicine, Medicine, \\ Department Of Medicine, Lokmanya Tilak Municipal Medical College and General Hospital, \\ Sion, Mumbai India.
}

\begin{abstract}
:
Background: HIV continues to be a major global public health issue. In 2014 an estimated 36.9 million people were living with HIV, a global prevalence of $0.8 \%{ }^{(1)}$ The clinical spectrum of HIV infection encompasses a spectrum ranging from an acute syndrome associated with primary infection to a prolonged symptomatic state to an advanced disease ${ }^{(2)}$ Cardiac involvement in HIV affected patients is becoming more prevalent as therapy and longevity improve Infection with HIV is one of the causes of acquired heart disease in these patients ${ }^{(3,4)}$.

Methods: This was an observational study, conducted in a tertiary care centre with an aim to study 2Echographic parameters, and its co-relationship with immunological status of the patient, as measured by CD4 counts in patients with HIV/AIDS.

Results: In our study 151 HIV positive patients were studied over a period of 12 months .Majority of our patients were in the age group of 20-40 years with average age of 38.7years. Male patients were $59.7 \%$ and $40.3 \%$ patients were females. Out of the 151 cases, $34.4 \%$ had CD4 count $<200,38.4 \%$ had CD4 count between 200-350 and 27.2\% had CD4 count $>350.25 .7 \%$ patients had some abnormal 2D echo findings which was associated with low CD4 count (<350). Among the echocadiographic findings $76.8 \%$ patients had normal LVEF (>60\%) and $23.2 \%$ had low LVEF (<60\%).

Conclusion: Echocardiographic abnormalities are more prevalent in HIV/AIDS patients and their prevalence increases as the CD4 count falls. So we should aim at starting ART early in HIV infected patients so as to improve The quality of life of people living with HIV/AIDS.

Keywords: HIV , 2D Echography, Pericardial Effusion.CD4.

\section{Introduction}

In 2015, there were 36.7 million [34.0 million-39.8 million] people living with HIV. 17 million people living with HIV were accessing antiretroviral therapy, up from 15.8 million in June 2015 and 7.5 million in 2010. In the United States, approximately $10 \%$ of those infected are older than 50 years and more than $85 \%$ of HIV-infected individuals survive more than 10 years $^{(1)}$. The 2- to-5-year incidence of symptomatic heart failure ranges from $4 \%$ to $28 \%$, suggesting a prevalence of symptomatic HIV-related heart failure of between 4 and 5 million cases worldwide ${ }^{(5)}$. The overall clinical spectrum of HIV disease is constantly changing with advent of HAART. Antiretroviral therapy clearly increases survival, and goals now involve balancing survival and toxicities with therapy ${ }^{(6)}$ With this view in mind we undertook the study of cardiac dysfunctions with the help of 2D echo findings in patients with HIV/AIDS with their clinical correlation.
\end{abstract}

\section{Material And Methods}

This was an observational study, conducted in a tertiary care centre with an aim 1 . To study $2 \mathrm{D}$ ECHO CARDIOGRAPHIC profile of patients with HIV/AIDS .

\section{To study clinical correlation between CD4 levels and Echocardiographic findings.}

The study was undertaken after clearance was obtained from the "Institutional Ethics Review Board". Patients were selected from both outdoor and indoor areas and were assessed for CD4 count, Haemoglobin levels, blood sugar levels (fasting, postprandial or random), liver function tests, renal function tests, lipid profile, serum electrolytes and Xray chest. Patients were asked for presence of any co-morbid condition like presence of diabetes mellitus, hypertension and ischemic heart disease. Vital parameters of all patients were noted on admission. Patients were thoroughly examined for signs of cardiac failure. A complete systemic examination was performed. Patients were advised 2D echocardiography and were asked to follow up with reports .The analysis of above mentioned study was done by using statistical methods. Depending on the interpretation and results ,prevalence of Echocardiography changes and its correlation with CD4 counts and their statistical significance was derived. 


\section{Results}

A total number of 151 cases were included in this study. Out of the 151 patients included 90(59.7\%) were males and 61(40.3\%) were females. Maximum patients ,97(64.7\%)were from the age group of 20-40 years ,the means age being 38.3 years. Fifty three (35.1\%)patients were more than 40 year and only 1 patient was less than 20 years. Out of the 151 patients included 52(34.4\%) had CD4 count <200, $58(38.4 \%)$ had CD4 count between 200-350 and $41(27.2 \%)$ had CD4 count $>350$. In this study $127(84.1 \%$ )patients were on HAART and $24(15.9 \%)$ were ART naive patients. The average duration of disease in patients on ART was 13.3 months (SD-17.7). Average duration of taking HAART was 9.8 months (SD -15.8). Out of the 151 patients studied $106(70.2 \%)$ had normal ECG findings and 45(29.8\%) had ECG abnormalities in the form of abnormal sinus rhythm, axis deviation or significant ST-T wave changes. The average CD4 count of patients with abnormal ECGs was 158 and average CD4 count of patients with normal ECG was 314.There was highly significant correlation between ECG abnormalities and low CD4 count with a P-value of $<0.0001$.(Figure- 1) In this study 97(64.3\%) had normal 2DEchocardiography, and $54(35.7 \%)$ had at laeast 1 abnormality detected in echocardiao graphy.

Out of 151 patients,116 (76.8\%) patients had normal LVEF (>60\%), 19 (12.6\%) patients had mildly decreased LVEF (60-45\%), 10 (6.7\%) had moderately decreased LVEF (45-30\%) and 6 (3.9\%) patients had severely decreased LVEF $(<30 \%)$. The average CD4 count of patients with normal LVEF was 310(SD-138) and average CD4 count of patients with low LVEF was 126(SD-72) ( Figure-2). In our study, 18(11.9\%) patients had pericardial effusion and $133(88.1 \%)$ did not have any pericardial effusion. Five (3.4\%) patients had thin rim of pericardial effusion, 3(1.9\%) patients had mild pericardial effusion, $9(5.9 \%)$ patients had moderate pericardial effusion and $1(0.7 \%)$ had severe pericardial effusion. The average CD4 count of patients with no pericardial effusion was 282(SD-149) and average CD4 count of patients with pericardial effusion was 159(SD71). This shows that there is a significant correlation between low CD4 $(<350)$ and presence of Pericardial effusion. The commonest aetiology of Pericardial effusion was Tuberculosis and patients were treated accordingly. Out of the 151 patients, 111 (73.6\%) patients had no diastolic dysfunction (DD) and 40 (26.4\%) patients had diastolic dysfunction. Nineteen (12.5\%) patients had type-1 DD and 21(13.9\%) had type-2 DD. The average CD4 count of patients with no diastolic dysfunction was 307(SD-148) and average CD4 count of patients with diastolic dysfunction was 157 (SD-68). This shows that there is a significant correlation between low CD4 (<350) and presence of Diastolic Dysfunction. The study showed that $15.9 \%$ of our patients had PAH. 9.9\% had mild PAH, 5.3\% had moderate PAH and 0.7\% had severe PAH. The average CD4 count of patients with no PAH was 284 (SD-151) and average CD4 count of patients with PAH was 176(SD-78). This shows that there is a significant correlation between low CD4 (<350) and presence of PAH.

\section{Discussion}

HIV compromises the immunity of a person to such a level that the person affected becomes highly vulnerable to variety of infectious and non-infectious diseases. For many years the infectious diseases were focused upon as they clinical spectrum of HIV disease is constantly changing with advent of HAART. Cardiac complications of HIV infection tend to occur late in the disease or are associated with related therapies. Thus cardiac involvement in HIV affected patients is becoming more prevalent as therapy and longevity improve. As survival improves, cardiovascular disease has become an increasingly important cause of morbidity and mortality among patients with HIV infection.

Our study included $59.7 \%$ males and $40.3 \%$ female and similar study by V.Chengat et all on 284 patients had $63.1 \%$ males and $36.9 \%$ females ${ }^{(8)}$. Average age in our study was 38.7 years and a similar study by Guha et al also had the average age of 33.24 years $^{(9)}$. Here 97(64.3\%) patients had normal 2D Echocardiographic findings and 54(35.7\%) had at least one abnormal finding in their Echocardiogram (LVEF $<60 \%$, Diastolic Dysfunction, Pericardial Effusion, Pulmonary Artery Hypertension). The average CD4 count of patients with normal 2D echocardiography was 333(SD 138) and the average CD4 count of patients with some abnormality in 2D Echocardiography was 149(SD 72) (Figure-3). Out of 151 patients,116(76.8\%) patients had normal LVEF (>60\%), 19(12.6\%) patients had mildly decreased LVEF (60-45\%), 10(6.7\%) had moderately decreased LVEF (45-30\%) and 6(3.9\%) patients had severely decreased LVEF (<30\%). In a study done by Twagirumukiza et al17\% had low $\mathrm{LVEF}^{(10)}$. 23.2\% of our patients had Regional Wall Motion Abnormality(RWMA). The average CD4 count of patients with no RWMA was 310(SD-138) and average CD4 of patients with RWMA was 126(SD-72). The correlation between presence of RWMA and low CD4 count $(<350)$ was statistically significant. In our study $11.9 \%$ patients had pericardial effusion. Most common aetiology was tuberculosis. Heidenrech et all had a prevalence of tuberculosis $5 \%$ and incidence of $11 \%$ in their study ${ }^{(11)}$. The average CD4 in patients was 284 (SD-149). There is significant correlation between presence of pericardial effusion and low CD4 count (<350). In our study $26.4 \%$ patients had Diastolic Dysfunction(DD).According to a study by Reinsch $\mathrm{N}$ et al the prevalence of Diastolic Dysfunction in HIV infected patients was $48 \%{ }^{(12)}$. The average CD4 of patients with no Diastolic Dysfunction was 307(SD-148) and 
the average CD4 of patients with diastolic Dysfunction was 157(SD-68) which was statistically significant. In a study by Chang et al LV systolic and Diastolic dysfunctions were positively correlated with decreased CD4 count ${ }^{(13)} .15 .9 \%$ of our patients had PAH. According to Quezada et al PAH was diagnosed in $9.9 \%$ patients ${ }^{(14)}$. The average CD4 in patients with no PAH was 284(SD-151) and in patients with PAH was 176(SD-78) which was significant.

\section{Conclusion}

Cardiac involvement is common amongst HIV/AIDS patients with $35.7 \%$ of our patients having cardiac involvement. Even asymptomatic patients had positive echocardiographic findings. Patients with longer duration of disease were more prone to have cardiac Involvement. Echocardiographic abnormalities are more prevalent in HIV/AIDS patients and their prevalence increases as the CD4 count falls. The tendency to develop these manifestations increases when CD4 is $<350$ which further increases as the counts further fall $<200$. Thus our aim should be to start ART in these patients as soon as possible so as to improve the morbidity and quality of life of people living with HIV/AIDS.

\section{Figures}

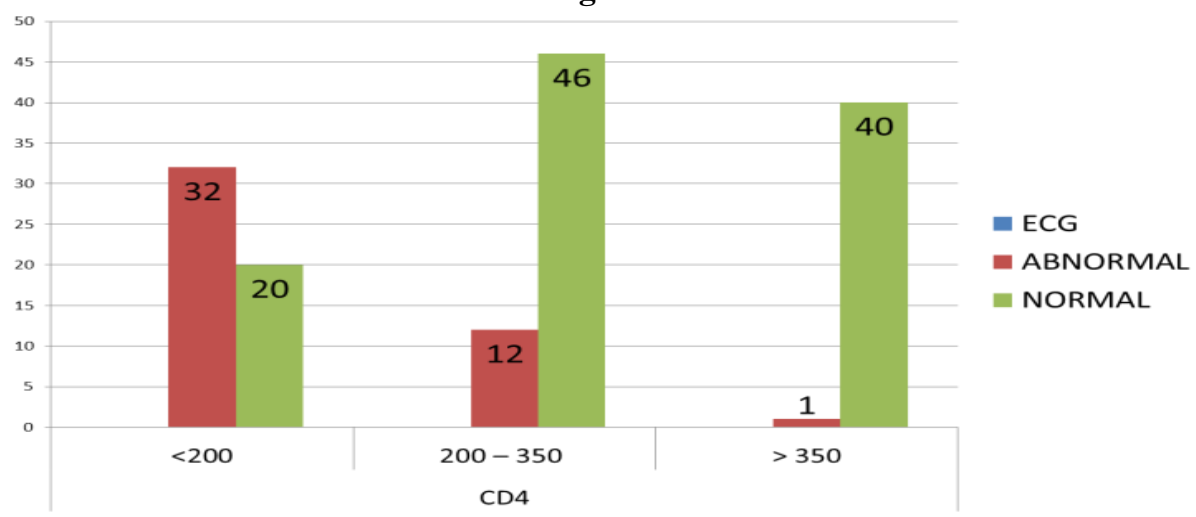

Figure 1: Correlation between Abnormal ECG findings and CD4 counts..

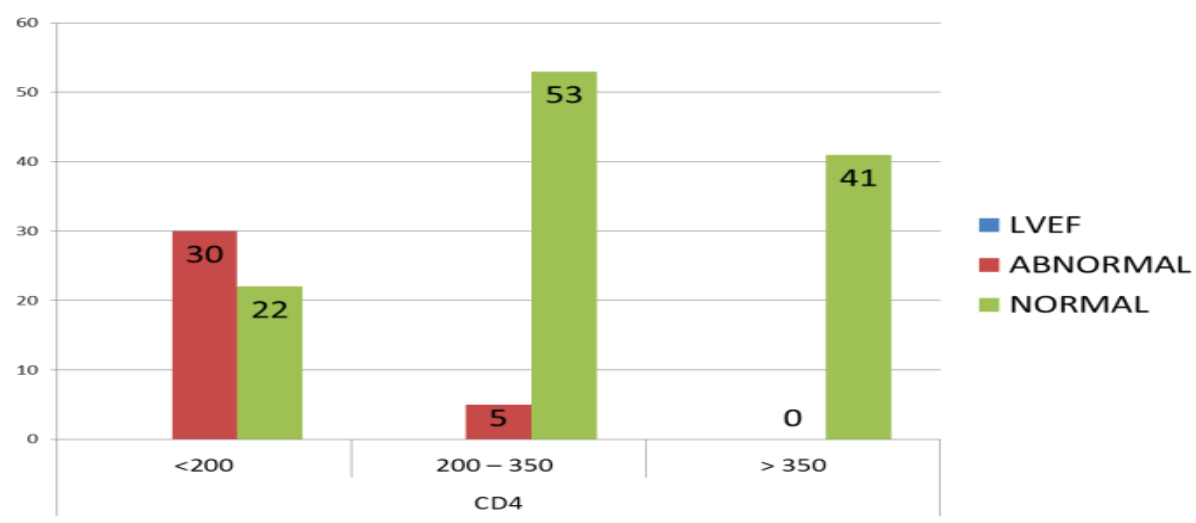

Figure 2: Correlation between LVEF and CD4 counts

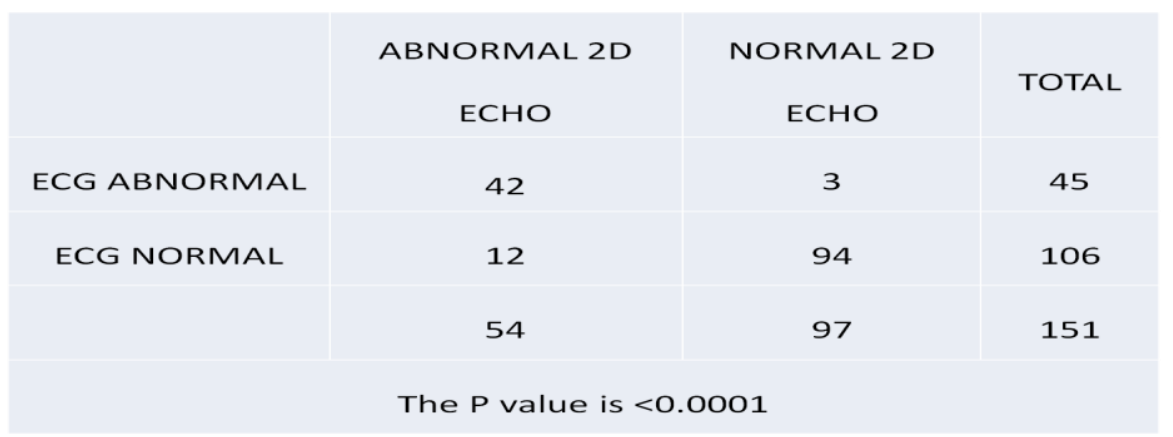

Figure 3: Correlation between Abnormal ECG and Abnormal 2DEcho 


\section{References}

[1]. Www.unaids.org/en/regionscountries/countries/india Epidemiological Fact Sheet on HIV, UNAIDS factsheet 2015.

[2]. Harrison's Principles of Internal Medicine $18^{\text {th }}$ edition, Chapter 189, Human Immunodeficiency Virus Disease: AIDS and Related Disorders.

[3]. Ho JE,Hsue PY:Cardiovascular Manifestations of HIV infections.Heart 2009;95:1193.

[4]. Barbaro G. HIV-associated cardiomyopathy etiopathogenesis and clinical aspects. Herz. 2005 Sep;30(6):486-92. Review. PubMed PMID: 16170679

[5]. Lipshultz SE, Fisher SD, Lai WW, Miller TL: Cardiovascular risk factors, monitoring, and therapy for HIV-infected patients. AIDS 2003; 17:S96.

[6]. Currie PF, Boon NA: Immunopathogenesis of HIV-related heart muscle disease: Current perspectives. AIDS 2003; 17:S21.

[7]. Sani MU. Myocardial disease in human immunodeficiency virus (HIV) infection: a review. Wien Klin Wochenschr. 2008;120(34):77-87. doi: 10.1007/s00508-008-0935-3. Review. PubMed PMID: 18322768Barbaro G. HIV-associated cardiomyopathy etiopathogenesis and clinical aspects. Herz. 2005 Sep;30(6):486-92. Review. PubMed PMID: 16170679.

[8]. V. Chengat, R. Subbiah, O. Raghunath, A. Rathinavel: Profile of HIV Associated Cardiomyopathy and Cardiac Isoform of Alpha Two Macroglobulin. The Internet Journal of Cardiology. 2010 Volume 8 Number 1. DOI: 10.5580/138c The study has been conducted in Madurai medical college and Maurai Kamraj University.

[9]. "Echocardiographic profile of ART naïve Human Immunodeficiency Virus (HIV) infected patients in a tertiary care hospital in Kolkata" Santanu Guha, Arindam Pande, Soura Mookerjee, Rabindra Bhattacharya, Shantasil Pain, Rathindra Nath Karmakar, Siddhartha Mani, Raja Bhattacharya, Anirban Biswas, Hema Sandip Saha, P.K. Deb, *Department of Cardiology, Department of Medicine Medical College Kolkata,

[10]. West Bengal ESI Hospital Maniktala , West Bengal; Indian Heart Journal 2010; july-aug-10 62:330-334

[11]. Twagirumukiza M, Nkeramihigo E, Seminega B, Gasakure E, Boccara F, Barbaro G. Prevalence of dilated cardiomyopathy in HIVinfected African patients not receiving HAART: a multicenter, observational, prospective, cohort study in Rwanda. Curr HIV Res. 2007 Jan;5(1):129-37. PubMed PMID: 17266564

[12]. Pericardial effusion in AIDS. Incidence and survival. Heidenreich PA, Eisenberg MJ, Kee LL, Somelofski CA, Hollander H, Schiller NB, Cheitlin MD. Circulation. 1995 Dec 1;92(11):3229-34.PMID:7586308

[13]. Reinsch N, Neuhaus K, Esser S, Potthoff A, Hower M, Brockmeyer NH, Erbel R, Neumann T; German Competence Network for Heart Failure; German Competence Network for HIV AIDS. Prevalence of cardiac diastolic dysfunction in HIV-infected patients: results of the HIV-HEART study. HIV Clin Trials. 2010 May-Jun;11(3):156-62. doi: 10.1310/hct1103-156. PubMed PMID: 20739268.

[14]. Chang WT, Wu CC, Hung CC, Chen MY,Faang CT, Chen Wj, Chaung CY, Lee YT. Left Ventricular dysfunction is associated with CD4 lymphocyte count rather than opportunistic infection in Human Immunodeficiency Virus infection.J Formos Med Assoc.2003 Mar;102(3):158-68.PubMed PMID:12783132.

[15]. Quezada M, Martin-Carbonero L, Soriano V, Vispo E, Valencia E, Moreno V, de Isla LP, Lennie V, Almería C, Zamorano JL. Prevalence and risk factors associated with pulmonary hypertension in HIV-infected patients on regular follow-up. AIDS. 2012 Jul 17;26(11):1387-92. doi:10.1097/QAD.0b013e328354f5a1. PubMed PMID:22526521 